\title{
NON-BRONCHOSPIROMETRIC MEASUREMENT OF DIFFERENTIAL LUNG FUNCTION

\author{
BY
}

\author{
G. H. ARMITAGE AND A. BRIAN TAYLOR \\ From the Department of Medicine, Queen Elizabeth Hospital, Birmingham
}

(RECEIVED FOR PUBLICATION MAY 14, 1956)

The introduction of bronchospirometry by Jacobacus in 1932, and subsequent improvements in the technique, have led to many valuable observations on the differential and relative function of the two lungs. The method, however, can be seriously misleading to those without long experience in its use, and Gaensler and Watson (1952) and Comroe and Kraffert (1950) regard it as a form of investigation primarily suitable for the larger thoracic centres.

Most of the difficulties of bronchospirometry for patient and for physician are contingent upon its principle of complete physical separation and measurement of the products of each lung's respiration. These objectives may be defeated by the presence of bronchial deformity, and the means of achieving them involves a degree of narrowing of the airway barely tolerable by the more dyspnoeic patients. The artificial separation of the airways, moreover, imposes on an underventilated lung the need to ventilate a larger dead space than under natural conditions, where the effect on each lung of the supracarinal dead space is in proportion to its share of the total ventilation. The original closed-circuit procedure involves high oxygen concentrations in the breathing-mixture which, as Gaensler has shown by his ambient-air method, may cause a material overestimate of the usual oxygen uptake of an underventilated lung.

The use has been suggested of differences in respiratory exchange ratio (R.Q.) between simultaneous samples of expired air from each main bronchus and trachea in order to calculate the share of each lung in oxygen uptake and ventilation (Armitage and Arnott, 1951). By this principle the main difficulties of bronchospirometry could be avoided and, at little cost to the convenience of the patient, results obtained comparable with those of bronchospirometry in value to a surgeon contemplating major surgery of lung or pleura. The R.Q. was selected as the index for comparison because it is unaltered by simple dilution of an expired air sample by any volume of air, and because when two sources of expired air mix the proportion of the oxygen deficit in the final mixture for which each is responsible may be calculated from the three R.Q. differences alone. That unilateral partial loss of function would give rise to an R.Q. difference between the two lungs seemed certain, since, just as generalized pulmonary disease invariably causes change in alveolar air composition, so must unilateral disease change the gas tensions within the affected lung ; and the exposure of the same mixed venous blood to alveolar air of differing $\mathrm{pO}_{2}$ and $\mathrm{pCO}_{2}$ in the two lungs must result in two distinct respiratory exchange ratios.

The present report is based on the results of 61 air samplings $(a)$ in a control group of 10 patients eventually shown to be without pulmonary disease, and $(b)$ in 49 patients with unilateral or symmetrical disease (two of them being reinvestigated some months later). Forty-seven of the resulting 51 sets of samples were suitable for determination of differential contributions.

The results will be considered primarily for their bearing on the practicability of the suggested procedure in determining differential function. The following conditions have to be satisfied. (1) Identical or almost identical R.Q.s should be found in both lung samples only when both lungs are normal, or when one is so severely damaged that total loss of function is a reasonable assumption. (2) Consistent substantial R.Q. differences should be found in unilateral disease insufficient to cause total loss of function. (3) Adequate mixing of expiration from both lungs must be attained below the points of tracheal sampling, as shown by absence of significant R.Q. differences between samples from two levels in the trachea (in patients showing material R.Q. differences between the two bronchial samples).

The validity of the calculation of differential oxygen uptake cannot be directly tested except by comparison with bronchospirometry in the same patient. Parallel bronchospirometric and airsampling studies are being carried out in a series of patients and the results will be separately reported ; 
in the first nine cases they have been in good agreement, the greatest difference between the two methods being $4 \%$, i.e., $34 \%$ of $\mathrm{O}_{2}$ uptake by diseased lung according to bronchospirometry, and $30 \%$ by the differential air-sampling technique (McIlveen and Armitage, unpublished observations). Here, only evidence of a more indirect kind will be presented. Partly this will take the form of details of patients later subjected to pneumonectomy or comparable major operative procedure, in whom the post-operative course could be expected to throw light on the accuracy or otherwise of the proportion of function attributed to the removed lung. A further indication will be the consistency with which the diseased lung was in fact calculated to have a subnormal share of total oxygen uptake (taking " normal" as $55 \%$ for the right lung and $45 \%$ for the left).

\section{Apparatus AND Methods}

The air samples are drawn into four oiled $50 \mathrm{ml}$ all-glass syringes mounted on a single carrier by spring clips. They may have conventional nozzles, which can be sealed by blank needle-hubs or be fitted with glass two-way taps. Most such syringes will store gas samples for 24 to 48 hours without detectable change in composition, but each should be checked in this respect.

The syringes are connected by lengths of "pige" P.V.C. tubing to the four catheters. These are of millimetre bore stainless steel needle tubing (Accles and Pollock). Two, for sampling from the main bronchi, are a few centimetres longer than the bronchoscope, that for the right bronchus being straight, the other slightly angled at the tip, and each with a lateral opening as near as possible to the tip, to minimize the risk of blockage. The remaining pair are of such lengths that, with their outer ends level with those of the bronchial catheters, their collecting ends will be 10 and $20 \mathrm{~cm}$. respectively above the carina. Some of the earlier air samples were taken continuously during inspiration and expiration, over a five-minute period. Samples were drawn into tonometers by the mechanical lowering of a common mercury reservoir. The method was cumbersome and the continuous sampling led to the dead space gas being sampled twice, once during expiration and again in inspiration. Probably as a result, such samples frequently showed a gradient of R.Q. falling as the point of sampling passed internally, so that instead of averaging the two tracheal R.Q. values, as at present, it was necessary to extrapolate to determine the theoretical tracheal R.Q. at the level of the carina.

AIR SAMPLING.-Air sampling is carried out at the end of routine bronchoscopy, under local anaesthesia. Excess secretion is sucked from the bronchi. The two bronchial catheters are inserted to a depth of not more than $2.5 \mathrm{~cm}$. The tracheal catheters are inserted into the bronchoscope to their correct level and sampling proceeds. The syringe holder is clamped or held by a nurse. Then, with two plungers grasped by each hand, one aspirates slowly during successive expirations (about $5 \mathrm{ml}$. during each cycle). The first 10 or $15 \mathrm{ml}$, which contain the gas from the of dead space of catheters and connexions, are rejected through each side tube, and sampling is continued till each syringe holds about $30-35 \mathrm{ml}$. of expired air $\vec{\omega}$ (to take more increases noticeably the rate of diffusion of air into the samples). The syringes are sealed, $\vec{x}$ and (after a final check on the position of the catheter tips) finally withdrawn.

By this method, with most of the dead space external to the sampling points, samples are usually $\infty$ obtained with about $80 \%$ of the $\mathrm{CO}_{2}$ concentration of true alveolar air.

GAS ANALYSIS.-All analyses should be done on one day, preferably the day the samples are taken. They should be accurate to $0.02 \% \mathrm{~N}_{2}$, or, in terms of R.Q., to $0.005-0.013$ according to the concentration of the sample.

All the analyses for the present study were performed by one of us, using a Sleigh apparatus, which gives the same repeatability in our hands as the Haldane $\left( \pm 0.01 \% \mathrm{~N}_{2}\right)$.

Method of Calculation.-The R.Q. of any sample of expired air is given by the formula

$$
R Q=\frac{\mathrm{CO}_{2} \%-0.03}{\left(20.93 \times \frac{\left.\mathrm{N}_{2} \%\right)}{79.04}-\mathrm{O}_{2} \%\right.}
$$

The calculation may be illustrated from an $\frac{0}{3}$ imaginary (but typical) example (Table I), in which the tracheal sample $T$ has the composition of a $3: 1$ F mixture by volume of the supposed right and left bronchial samples $\mathbf{R}$ and $\mathbf{L}$. Thus the situation represented is one where the right lung contributes 0.75 of the ventilation, and (in consequence of the larger $\mathrm{O}_{2}$ deficit on the right) $0.75 \times \frac{5.17}{3.80}$ or 0.803 of the total $\mathrm{O}_{2}$ uptake. The latter contribution-fraction is given by the following equation, from the R.Q. differences.

$$
\begin{aligned}
\frac{\text { Right }}{\text { Total }} \mathrm{O}_{2} \text { uptake } & =\frac{\mathrm{RQ}_{\mathrm{L}}-\mathrm{RQ}_{\mathrm{T}}}{\mathrm{RQ}_{\mathbf{L}}-\mathrm{RQ}_{\mathrm{R}}} \\
& =\frac{0.913-0.797}{0.913-0.768} \\
& =0.800
\end{aligned}
$$

TABLE I

\begin{tabular}{c|c|c|c|c|c}
\hline \multicolumn{5}{c}{ Percentages } \\
\hline Sample & $\mathrm{CO}_{2}$ & $\mathrm{O}_{2}$ & $\mathrm{~N}_{2}$ & $\mathrm{O}_{2}$ Deficit & R.Q. \\
\hline $\mathbf{R}$ & 4.00 & 16.00 & 80.00 & 5.17 & $\mathbf{0 . 7 6 8}$ \\
$\mathbf{L}$ & 3.50 & 17.20 & 79.30 & 3.80 & $\mathbf{0 . 9 1 3}$ \\
T & 3.88 & 16.30 & 79.82 & 4.83 & $\mathbf{0 . 7 9 7}$ \\
& & & & & \\
\hline
\end{tabular}

The relative contribution to ventilation can be calculated by substituting for the R.Q. figures in the 
equation the values from whichever of the other four columns of Table I shows the largest differences. This calculation, however, can be falsified by contamination of any or all of the samples with air during or after sampling (which would not affect the R.Q. values); moreover, since the ventilation involved in the calculation is not the true alveolar ventilation, but simply the air-flow past the sampling points, the calculation is of little value if the relative oxygen consumptions are known.

\section{Results and Analysis of Data}

\section{R.Q. Differences Between Apparently} Healthy Lungs. - Ten patients were studied. Two were being bronchoscoped for removal of inhaled foreign bodies (which had caused neither collapse nor localized inflammation) and in the remainder the purpose of bronchoscopy (which showed no abnormality) was to exclude lung disease. The clinical contexts included supposed haemoptyses, intracranial tumour (? primary), suspected carcinomatosis, etc. In all, the absence of lung disease was finally established.

The mean R.Q. difference between healthy lungs was 0.012 ; S.D. 0.005 , S.E. of mean 0.003 , range $0.003-0.021$. There was no tendency for either lung to show the higher R.Q.; taking account of sign, the mean right-left R.Q. difference was +0.001 .

R.Q. Differences Between UpPeR and LoWer Tracheal SAMPLES.-These results demonstrate the completeness of mixing of expiratory streams $10 \mathrm{~cm}$. above the carina in cases with significant inter-lung R.Q. differences. Because of the artificial tracheal R.Q. gradient produced by the original continuous-aspiration method of sampling, cases thus studied have been excluded.

Twenty-three patients were studied, with mean R.-L. R.Q. differences of 0.156 (S.D. 0.129 ; S.E. of mean 0.027 ).

The mean R.Q. difference between the upper and lower tracheal samples was 0.009 ; S.D. 0.007 ; S.E. of mean 0.001 , range $0-0.021$.

These two groups of observations establish two of the basic postulates of the method. First, the maximum R.Q. difference between normal lungs was 0.021 (mean 0.012). Secondly, the same maximum difference and an even lower mean was found between samples from two tracheal levels in 23 patients with very considerable inter-lung differences ; evidently, at $10 \mathrm{~cm}$. above the carina the expiratory streams are fully mixed.

Differential Function.-Four of the 51 studies were unsuccessful. In one a distorted main bronchus could not be catheterized. In another (later successfully repeated) the catheter was inserted too far into the left main bronchus, past the origin of the upper lobe division. The other two failures were in asthmatic patients with emphysematous bullae; the tracheal samples (taken only 5 and $10 \mathrm{~cm}$. above the carina very early in the series) differed widely in composition, each closely resembling one or other bronchial sample, indicating the presence of two expiratory streams still unmixed at 5 and $10 \mathrm{~cm}$. above the carina. (Both patients had considerable bronchospasm and prolonged expiration.)

Non-function of Diseased Lung.-R.Q. values identical within 0.020 in both bronchial samples were found in 10 patients (and confirmed in one on re-examination five months later). In all, this was taken to mean that the diseased lung had lost all respiratory function.

It was realized when this method was first conceived that the R.Q. difference created by impairment of function of one lung would disappear once all alveolar ventilation had been lost, for then the main bronchus of the affected side would become no more than a tidal backwater to the respiratory exchange of the normal lung, contributing nothing of its own. Under such circumstances virtual identity of R.Q.s would be restored.

Thus, though the method of differential air sampling can distinguish all the intermediate degrees of functional asymmetry, its results must be ambiguous at the two extremes of bilateral normal function on the one hand and total unilateral loss of function on the other. Empirical choice between these alternatives is in practice not difficult. However, if it is necessary to justify the intrusion of an element of choice into a supposedly quantitative technique, a precedent may be found in the traditional 12-hour clock whose inability to tell night from day is rarely a source of confusion in practice. The ambiguity of the differential sampling technique is less, since it only requires a supplementary subjective judgment at noon, so to speak, and at midnight.

This is illustrated by the findings in the 11 studies where identity of bronchial R.Q. values was taken to mean the presence of only one functioning lung. In one patient with a lung abscess unilateral absence of function was confirmed by conventional bronchospirometry; in another with extensive pulmonary tuberculosis the procedure was repeated after several months and some clinical improvement, with the same result. Subsequent pneumonectomy was well tolerated, and brought no increase in dyspnoea. The upper and middle lobes were found to be virtually destroyed by widespread disease: the lower lobe was 
bound by dense adhesions. A third patient eventually had a pneumonectomy for carcinoma of the left main bronchus, which had presented with acute dyspnoea: air trapping had preserved the relatively normal radiological appearance, but on the results of the sampling, supported by the clinical evidence of poor air entry, operation was undertaken, with great relief of dyspnoea. The lung would not collapse after removal because of the valvular nature of the bronchial obstruction. Without the evidence of air sampling in the latter two cases operation would on balance have been contraindicated by the clinical and radiological findings and the poor results of function tests. Two bronchiectatic patients who also underwent pneumonectomy gave the clinical and radiological impression of unilateral absence of function; in them operation might well have been undertaken even without the confirmation afforded by the air sampling.

Thus in these five cases the non-function of the diseased lung suggested by the air sampling was confirmed or supported either by conventional bronchospirometry or, on subsequent pneumonectomy, by the appearance of the lung and the effect of its removal on the pre-existing severe dyspnoea. In the remaining five patients, very severe or complete loss of function had been expected, on clinical and radiological evidence, before the air sampling added the evidence of identity of R.Q. in both bronchial samples. Although none of the 10 cases had evidence of substantial disease of the "normal" lung, their vital capacities and (in five) maximum breathing capacities were greatly reduced, ranging respectively from $48 \%$ to $70 \%$ (mean $57 \%$ ) and from $38 \%$ to $66 \%$ (mean $53 \%$ ) of the values predicted from the formulae of Baldwin, Cournand, and Richards (1948). Pneumonectomy, in the face of such severe reductions in functional capacity, might well have seemed too hazardous without the reasonable certainty that the lungs to be removed were functionless.

Unequal Function of the Two Lungs.-Thirtysix studies in 35 patients showed significant R.Q. differences between the right and left bronchial samples, indicating the retention of some function by the affected lung.

In 14 patients, in none of whom was the sounder lung completely normal, the practicability of a projected pneumonectomy or lesser procedure was in question. Of these, eight had a calculated share in function by the diseased lung so small as to be negligible, and all underwent operation without subsequent increase in dyspnoea, while in the remaining six the calculation showed a proportion of remaining function greater than could be $\frac{\bar{\sigma}}{\bar{\omega}}$ sacrificed or prejudiced with safety. In only one $\overrightarrow{\widetilde{\Phi}}$

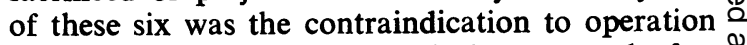
ignored at the patient's own insistence, and after के pneumonectomy for severe left bronchiectasis $\overrightarrow{0}$ (with asthma and emphysema) this patient was $\overrightarrow{-}$ unable for four weeks to leave the oxygen tent, $\vec{\sigma}$ and died after a further month with a middle lobe pneumonia. The air sampling test had shown $\stackrel{x}{.}$ $22 \%$ of oxygen uptake from the lung which was later removed, a contribution which, in the presence of dyspnoea on the least exertion, and $\stackrel{N}{\rightarrow}$ proven pulmonary hypertension, it had been felt $\vec{\circ}$ dangerous to sacrifice. This was, however, one of the earliest patients of the series, and the reliability $\vec{\square}$ of the test was then problematical.

Thirty-four of the 36 air samplings indicated $\stackrel{\mathbb{}}{3}$ subnormal proportions of oxygen uptake by the lungs judged the more severely diseased clinically and radiologically. Normal shares are taken as $\vec{\oplus}$ $45 \%$ and $55 \%$ for left and right lungs respectively. I Both the exceptions were middle-aged men with emphysema and a single giant bullous cyst occupying a third to a quarter of the left lung. In the first that lung was calculated to have exactly its normal $45 \%$, while in the other the proportion $\frac{2}{\square}$ was as high as $58 \%$ and that of ventilation higher $\underset{\vec{P}}{\overrightarrow{2}}$

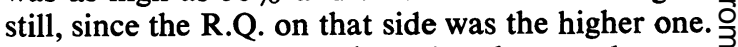
Fortunately this patient three days later underwent $\vec{\partial}$ bronchospirometry, which confirmed the air sampling result, attributing $55 \%$ of the oxygen uptake and $57 \%$ of the ventilation to the cystic lung.

In the 36 instances of asymmetrical function, $\underset{x}{\stackrel{0}{x}}$ the mean R.Q. difference of the bronchial samples was 0.167 ; S.D. 0.103 , S.E. of mean 0.017 , range 3 . 0.026-0.459. Two cases had inter-lung R.Q. differences of only 0.026 outside the range of $₹$ normal inter-lung and inter-tracheal differences, 을 but obviously too small for reliable calculation of $D$ functional contribution. One of these had severe을. post-empyema pleural thickening and total loss of function had seemed likely; the other had onlyo a minimal tuberculous scar at the left apex. The $N$ two calculated contributions were $4 \%$ and $39 \%$ స్ట respectively - reasonable in each case-but with sosmall a total difference they might well have been regarded as cases of identical R.Q.s, in the first case $\frac{\mathbb{\Phi}}{\mathbb{N}}$ from function being unilateral only, in the second $\stackrel{\oplus}{+}$ from it being normal on each side. The three 0 next smallest R.Q. differences were $0.058,0.060$, and 0.070 .

Two factors influence the magnitude of the $\frac{\mathbb{Q}}{\Omega}$ R.Q. difference. Disease which principallyo reduces ventilation lowers the R.Q. on the 
affected side, but cannot depress it below the "venous R.Q.," i.e., that of alveolar air in equilibrium with mixed venous blood, normally between 0.4 and 0.5 . There is no ceiling, however, to the possible rise in R.Q. when the disease primarily reduces perfusion. Thus, the mean R.Q. difference in the 16 patients with a higher R.Q. on the affected side was 0.197 ; in the remaining 20 with a lower R.Q. it was only 0.143 . The extent of loss of function also appears to have some bearing on the magnitude of the R.Q. difference, as Table II shows. In each group minor $(<1 / 3)$ loss of

TABLE II

RELATION BETWEEN MAGNITUDE OF R.O. DIFFERENCE AND DEGREE OF FUNCTION LOSS

\begin{tabular}{|c|c|c|c|c|}
\hline \multirow{2}{*}{$\begin{array}{l}\text { Function } \\
\text { Retained } \\
\text { (\% of Normal } \\
\text { Share) })^{*}\end{array}$} & \multicolumn{2}{|c|}{$\begin{array}{l}\text { Underperfused } \\
\text { (High R.Q.) Group }\end{array}$} & \multicolumn{2}{|c|}{$\begin{array}{l}\text { Underventilated } \\
\text { (Low R.Q.) Group }\end{array}$} \\
\hline & $\begin{array}{l}\text { No. of } \\
\text { Cases }\end{array}$ & $\begin{array}{c}\text { Mean } \\
\text { R.Q. } \\
\text { Difference }\end{array}$ & $\begin{array}{l}\text { No. of } \\
\text { Cases }\end{array}$ & $\begin{array}{c}\text { Mean } \\
\text { R.Q. } \\
\text { Difference }\end{array}$ \\
\hline $\begin{array}{r}1-33 \\
34-66 \\
67-99\end{array}$ & $\begin{array}{l}3 \\
7 \\
6\end{array}$ & $\begin{array}{l}0.212 \\
0.237 \\
0.141\end{array}$ & $\begin{array}{l}8 \\
7 \\
5\end{array}$ & $\begin{array}{l}0 \cdot 110 \\
0 \cdot 198 \\
0 \cdot 118\end{array}$ \\
\hline
\end{tabular}

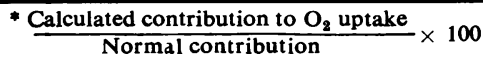

function is associated with relatively small R.Q. differences, and as function loss increases the R.Q. differences widen. However, in the primarily underventilated group, as function loss increases still further, the differences diminish strikingly. There were only a few individual exceptions to the trends illustrated by these averages.

It is likely, even in the low R.Q. group, that the true alveolar R.Q. of the affected lung is displaced increasingly, down to the point where alveolar ventilation and respiratory exchange cease, and the venous R.Q. is reached. In the bronchial samples, however, the R.Q. differences apparently diminish, as Table III shows, when loss of function has increased beyond a certain point. A possible explanation is that reduced tidal ventilation (even when some alveolar ventilation persists) may fail to clear from the intrapulmonary dead space the mixed alveolar air of both lungs inspired from the trachea at the beginning of inspiration. This will be sampled, along with the true alveolar ventilation component, during the next expiration and will mask the true extent of the R.Q. difference between the alveolar air of the two sides. With uniformly reduced ventilation such a mechanism would operate only just before all function was lost, whereas regional inequality of underventilation might bring it into play rather earlier, but with weaker effect. The differential calculation need not be affected, since an apparent reduction in the R.Q. difference between the affected lung and the trachea can be balanced by a much smaller change in the difference between tracheal and normal lung samples. In any case no error could arise sufficient to change an obviously negligible contribution into one apparently worth preserving.

Incidence of Low- and High-R.Q. Patterns in Unilateral Function Loss.-Gaensler, Watson, and Patton (1953) concluded from 1,069 spirometric studies that during every process of unilateral reduction in lung function-and especially those (the severest) resulting from bronchiectasis and from pleural disease-ventilation suffers a lesser and later decline than does oxygen consumption. The disproportion in the effect on the two functions was least, and often negligible, in disease of other aetiology, which usually caused relatively minor reduction in either function.

Since the R.Q. varies as the ventilation-perfusion ratio, and oxygen uptake (from the oxygenenriched mixtures employed in bronchospirometry) is a measure of perfusion, the above would imply a higher R.Q. on the affected side, especially in cases of pleural or bronchiectatic origin. However, in 20 of the 36 instances with significant R.Q. differences between the bronchial samples, the R.Q. was lower on the diseased side indicating relative underventilation, and in these 20 the average function loss was greater $(57 \%)$ than in the minority of 16 cases ( $44 \%$ ) with a high R.Q. Again, out of 14 patients with pleural or bronchiectatic disease, 11 had a lower R.Q. on the affected side.

The origin of this apparent contradiction lies in the inability of bronchospirometry to distinguish between alveolar and dead space ventilation. As ventilation is reduced, an increasing proportion is absorbed by the dead space, and, if this is allowed for, the relative impact of the disease on alveolar ventilation and oxygen consumption may appear in a very different light, as the following illustration shows.

Consider a case of moderate asymmetry of function, with a tidal ventilation of $600 \mathrm{ml}$. distributed 200:400 ml. to affected and normal lungs respectively; and with $\mathrm{O}_{2}$ uptake divided $30 \%$ : $70 \%$ between the two, and therefore in somewhat greater imbalance than the ventilation. The deduction from each lung's ventilation of $80 \mathrm{ml}$. for the dead space leaves the $440 \mathrm{ml}$. alveolar ventilation divided $120: 320$, or $27 \%: 73 \%$ between them. In other words, the affected lung's share of alveolar ventilation is as much less than its share of $\mathrm{O}_{2}$ uptake as the latter is less than its share of total ventilation. Were the same total ventilation to be 
divided $120: 480 \mathrm{ml}$, or $20 \%: 80 \%$, the dead space deduction would have an even greater relative effect, leaving alveolar ventilation divided 40:400 ml. or $9 \%: 91 \%$.

This point has been treated at some length because it illustrates how the air sampling technique can reveal and bronchospirometry obscure the fact that the commonest immediate mechanism of unilateral loss of respiratory function is through the development of alveolar underventilation. This has more than theoretical importance because (as the low R.Q. indicates) oxygen uptake from such a lung is accomplished only at the cost of $\mathrm{CO}_{2}$ retention and even of some arterial unsaturation. Nor will this veno-arterial shunt effect necessarily diminish when all respiratory function has finally been lost. Other things being equal, any contribution by a high-R.Q. lung is for this reason more likely to be worth preserving than the same contribution by a lung primarily underventilated.

Results According to Aetiology.-An attempt has been made in Table III to classify the results of the differential sampling according to aetiology. Not all the cases lent themselves to aetiological classification and it was not to be expected that the four broad groups would show very consistent or specific patterns of function loss. The groups were as follows :

I. Pulmonary tuberculosis $(a)$ without important pleural involvement; (b) pleural.

\section{Bronchiectasis.}

III. Bronchial carcinoma and atelectasis of other origin.

IV. Cystic disease, localized emphysema, etc.

Each patient was allocated to a group before the results of the sampling were known. In cases of multiple aetiology the most important, as judged clinically and radiologically, determined the diagnostic grouping. Group Ia contains only cases without evidence of material pleural involvement. Group Ib includes one case of pleural thickening of non-tuberculous origin. One of the patients twice investigated appears twice in Table III, since the second study followed the excision of a giant cyst and a change in the nature and amount of the affected lung's contribution to function.

As Table III shows, in Groups I and II (tuberculous and bronchiectatic) low R.Q. values predominated on the affected side, indicating relative underventilation. Group III (carcinoma and atelectasis) was equally divided. In Group IV
TABLE III

SUMMARY OF RESULTS IN FOUR MAIN AETIOLOGICAL GROUPS

\begin{tabular}{|c|c|c|c|c|}
\hline \multirow{3}{*}{ Group } & \multirow{3}{*}{$\begin{array}{l}\text { No. of } \\
\text { "One- } \\
\text { lung } \\
\text { Cases " }\end{array}$} & \multicolumn{3}{|c|}{ Bilateral but Unequal Function } \\
\hline & & \multicolumn{2}{|c|}{ Affected Lung R.Q. } & \multirow{2}{*}{$\begin{array}{c}\text { Mean Percentage of } \\
\text { Normal O Uptake } \\
\text { Share Retained }\end{array}$} \\
\hline & & Higher & Lower & \\
\hline $\begin{array}{l}\text { Ia } \\
\text { Ib } \\
\text { II } \\
\text { III } \\
\text { IV }\end{array}$ & $\begin{array}{l}3 \\
3 \\
3 \\
1\end{array}$ & $\begin{array}{l}1 \\
1 \\
2 \\
4 \\
8\end{array}$ & $\begin{array}{l}3 \\
4 \\
6 \\
4 \\
3\end{array}$ & $\begin{array}{r}56\} \\
33\} \\
37 \\
58 \\
52\end{array}$ \\
\hline Total & 10 & 16 & 20 & \\
\hline
\end{tabular}

(cystic disease, emphysema, emphysematous bullae, etc.) a higher R.Q. on the affected side was found in eight out of 11 cases. This indicated not necessarily an absolute hyperventilation of the affected lung (though in two or three cases this seemed likely) but that the ventilation was mainly directed to alveoli insufficiently perfused to take advantage of it.

\section{SUMMARY}

A method is described of measuring the function of each lung differentially, indicating both the ventilation and perfusion.

At the end of bronchoscopy under local anaes- $\frac{\partial}{\not}$ thesia air samples are taken during expiration $\unrhd$ from within each main bronchus and at two levels $\overrightarrow{\overrightarrow{0}}$ in the trachea. Estimations of the respiratory quotient (R.Q.) from analysis of these samples show the difference in function of each lung.

The advantages to the patient by the simplicity of the procedure, the greater information by avoiding oxygen breathing, and the accuracy of the $x$ results are demonstrated.

The practical advantages of assessing individual lung function before considering extensive thoracic surgical procedures are particularly emphasized in view of the comparative simplicity of the procedure.

We acknowledge our indebtedness to Professor W. Melville Arnott, Mr. A. L. d'Abreu, and Mr. o J. L. Collis for referring patients for investigation, and $N$ to the latter two for some of the bronchoscopies. Dr. N Donald McIlveen carried out bronchoscopy and $\omega$ bronchospirometry on nine of the patients.

\section{REFERENCES}

Armitage, G. H., and Arnott, W. M. (1951). J. Physiol. (Lond.), $\mathscr{\mathbb { D }}$ $112,23 P$.

Baldwin, E. de F., Cournand, A., and Richards, D. W., Jr. (1948). Medicine (Baltimore), $27,243$.

Comroe, J. H., and Kraffert, N. H. (1950). In Meth. med. Res., 2, 94. 으

Gaensler, E. A., and Watson, T. R. (1952). J. Lab. clin. Med., 40, 223.

$-1-\frac{23 .}{1}$ and Patton, W. E. (1953). Ibid., 41, 436.

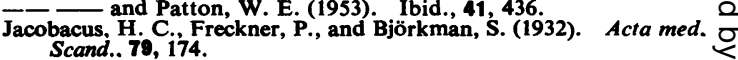

McIlveen, D., and Armitage, G. H. Unpublished data. 